\title{
Efficacy of sonic-powered
}

\section{toothbrushes for plaque removal in patients with peri-implant mucositis}

\author{
Jungwon Lee', Jong heun Lim², Jungeun Lee', Sungtae Kim', Ki-Tae Koo', \\ Yang-Jo Seol', Young Ku', Yong-Moo Lee', In-Chul Rhyu',* \\ 'Department of Periodontology, Seoul National University School of Dentistry, Seoul, Korea \\ ${ }^{2}$ The Armed Forces Medical Command, Seongnam, Korea
}

Purpose: The aim of this study was to evaluate the effectiveness of powered toothbrushes for plaque control in patients with peri-implant mucositis, in comparison with manual toothbrushes.

Methods: This randomized, prospective, controlled, clinical parallel study compared the efficacy of manual and powered toothbrushes for plaque control in implant restorations. Patients with bleeding on probing, no residual pocket depth (as indicated by a pocket probing depth $\geq 5 \mathrm{~mm}$ ), and no radiological peri-implant bone loss were eligible for this study. Patients were requested to complete a questionnaire describing their oral hygiene habits. The duration and frequency of tooth brushing were recorded by subjects in order to assess their compliance. Clinical parameters, including the modified plaque index (mPI), the modified sulcus bleeding index (mSBI), and clinical photographs (buccal and lingual views) were recorded at baseline and at one-month and two-month follow-up visits.

Results: Statistically significant differences between patients who used manual toothbrushes and those who used powered toothbrushes were found regarding the frequency of tooth brushing per day and the duration of brushing at one-month and two-month follow-up visits, while no statistically significant differences were found relating to other oral hygiene habits. A statistically significant difference in patient compliance for tooth brushing was found at one month, while no difference was found at two months. Statistically significant decreases in the $\mathrm{mPI}$ and the $\mathrm{mSBI}$ were observed in both groups from baseline to the one- and two-month follow-ups. The overall reduction of these parameters was not significantly different between the two groups, except for $\mathrm{mPI}$ reduction between baseline and one month of follow-up.

Conclusions: Sonic-powered toothbrushes may be a useful device for plaque control in patients with peri-implant mucositis.

Keywords: Dental implant, Dental plaque, Oral hygiene.

\section{INTRODUCTION}

Peri-implant mucositis is defined as inflammation in the mucosa around a dental implant. Gingivitis and peri-implant mucositis are similar in that both are reversible [1]. Early intervention with effective removal of the microbial biofilm is known to be helpful in preventing further progression to periodontitis and peri-implantitis, respectively [2]. Peri-implantitis is diagnosed when the inflamed mucosa is accompanied with bone loss around the dental implant [3]. If peri-implant mucositis is left untreated, it can result in the progressive destruction of the marginal bone around the dental implant, which in turn leads

This is an Open Access article distributed under the terms of the Creative Commons Attribution Non-Commercial License (http://creativecommons.org/licenses/by-nc/3.0/). 
to peri-implantitis $[4,5]$.

The presence of plaque around dental implants appears to be associated with peri-implant mucositis [6]. Peri-implant lesions are associated with inadequate plaque control at implant sites, whereas peri-implantitis is rare when proper plaque control is ensured $[7,8]$. In a long-term clinical study, peri-implant lesions were frequently found in patients who did not adhere to supportive therapy, including the removal of bacterial biofilm [6]. Most practitioners agree that the long-term success of implant restorations can be improved by maintaining good oral hygiene and preventing plaque accumulation around the implants $[9,10]$.

Daily plaque control using a manual toothbrush is one effective way to prevent plaque accumulation around teeth [11]. Dental floss and interdental cleansing devices are also recommended for daily plaque control. In some clinical situations, in which an implantsupported prosthesis does not have the proper contour, angulation, or location, more time and effort are required for the daily removal of plaque [12]. Powered toothbrushes may assist in maintaining good oral hygiene, as they provide patients with an easy plaque control technique that does not require substantial training [13].

Powered toothbrushes have advantages compared to other oral hygiene appliances. Sicilia et al. [14] reported that the use of powered toothbrushes, especially those with counter-rotational and oscillating-rotating brushes, can be beneficial in reducing the levels of gingival bleeding or inflammation. Vandekerckhove et al. [13] reported that powered toothbrushes are effective, safe and comfortable for patients who have undergone rehabilitation with implant prostheses. Wolff et al. [15] demonstrated that sonic brushing is an effective means of dental implant maintenance. On the other hand, Swierkot et al. [16] found no significant differences between manual and sonic-powered toothbrushes regarding plaque removal around dental implants. These results were obtained from subjects without periodontal or peri-implant disease. To the best of our knowledge, no studies have evaluated the effectiveness of sonicpowered toothbrushes for plaque removal in subjects with peri-implant mucositis.

The aim of this study was to evaluate the effectiveness of powered toothbrushes, in comparison with manual toothbrushes, for plaque control in patients with peri-implant mucositis.

\section{MATERIALS AND METHODS}

\section{Experimental design}

This randomized, prospective, controlled, clinical parallel study compared the efficacy of manual toothbrushes (Butler GUM 311, Sunstar, Etoy, Switzerland) and sonic-powered toothbrushes (Sonicare DiamondClean, Philips, Amsterdam, Netherland) for plaque control around dental implants. The sonic-powered toothbrush used in this study has a frequency of up to 31,000 brush strokes per minute and a two-minute timer to ensure the recommended brushing time. The study protocol was approved by the Institutional Review Board (IRB \#CMP13003), Seoul National University Dental Hospital,
Seoul, Korea. All patients received a detailed description of the proposed study protocol and provided informed written consent to participate in the study. Subjects were recruited from December 2013 to August 2014. The first procedure was carried out in December 2013, and all one-month follow-up visits were completed by October 2014. A block randomization sequence was used to ensure equal distribution of the subjects to the manual toothbrush group and the sonic-powered toothbrush group. The randomization was performed on the day of scaling, using a sealed envelope containing the allocated toothbrush procedure.

The sample size was determined to be 20 subjects per group. Wolff et al. [15] demonstrated that a change of $0.1( \pm 0.1)$ in the modified sulcus bleeding index (mSBI) around the implant sites was found between baseline and 24 weeks of follow-up. In our study, a difference of 0.3 in the magnitude of $\mathrm{mSBI}$ change after two months was considered to be the threshold of clinical relevance when comparing the use of manual and sonic-powered toothbrushes. A total sample size of 36 subjects (18 per group) was found to achieve $81 \%$ power in detecting a difference of 0.3 between the mean $\mathrm{mSBI}$ values of the manual toothbrush group and the sonic-powered toothbrush group, with a standard deviation of 0.3 . The Mann-Whitney $U$ test was used, and $P$-values $<0.05$ were considered to indicate statistical significance. Assuming a drop-out rate of $10 \%$, a total sample size of 40 (20 per group) was required.

\section{Population screening}

Patients with bleeding on probing, no residual pocket depth (as shown by a pocket probing depth $\geq 5 \mathrm{~mm}$ ), and no radiological peri-implant bone loss were considered eligible for this study. In order to be eligible for enrollment, all the patients had to meet the following inclusion and exclusion criteria.

The inclusion criteria of this study were: 1) the presence of at least one implant restoration, 2) no use of antibiotic medications within the previous three months, 3) the presence of an implant prosthesis that was installed at least three months previously, and 4) no peri-implant bone loss as shown by radiography.

The exclusion criteria of this study were: 1) pregnancy, being less than three months postpartum, bleeding disorders, hemophilia, diabetes, immunodeficiency, epilepsy, rheumatic heart disease, or a joint replacement prosthesis; 2) the use of cyclosporin, dilantin, calcium channel blockers, or the chronic use of non-steroidal antiinflammatory drugs; 3 ) the requirement of dental treatment due to dental caries, abscesses, or similar conditions; 4) the presence of an orthodontic appliance; and 5) the presence of a condition that would prevent a patient from actively controlling oral hygiene, such as Parkinson's disease or mental retardation.

\section{Procedures}

All subjects were instructed to brush their teeth and implants three times a day for two minutes. Subjects in the manual toothbrush group were instructed to brush their teeth and implants using the Bass technique [17], and subjects in the sonic-powered tooth- 
brush group were told to follow the manufacturer's instructions.

Clinical baseline measurements were taken at the screening appointment, along with a standard periapical radiograph in order to exclude peri-implantitis. The data included the modified plaque in$\operatorname{dex}(\mathrm{mPI}), \mathrm{mSBI}$ [18], and clinical photographs (buccal and lingual views). Clinical measurements were taken by blind and calibrated investigators. Scaling was performed to minimize the effect of natural teeth adjacent to the implant at the first visit. The data were uploaded to a database in which patient privacy was protected according to current regulations. Clinical parameters were recorded at baseline as well as at one-month and two-month follow-up visits.

Patients were requested to complete a questionnaire that assessed oral hygiene habits and patient compliance. The duration and frequency of tooth brushing were recorded by the subjects for compliance assessment.

\section{Statistical analysis}

After a brief training, two examiners (JWL, JEL) performed repeated measurements of the $\mathrm{mPI}$ and $\mathrm{mSBI}$ using clinical photographs one week apart. The intra-examiner reproducibility and inter-examiner reliability of the measurements of the $\mathrm{mPI}$ and $\mathrm{mSBI}$ were assessed using the intraclass correlation coefficient and Kappa statistics $[19,20]$.

The Student's t-test (for continuous measures) or the Mann-Whitney $U$ test (for categorical measures) was used to evaluate whether any statistically significant differences were present between the two groups at each time point, and the Wilcoxon signed-rank test was used to evaluate whether statistically significant changes occurred from baseline within each group. $P$-values $<0.05$ were considered statistically significant. Correlations between the age of subjects and the change of the $\mathrm{mPI}$ and $\mathrm{mSBI}$ were determined with the Spearman's correlation test. All statistical analyses were performed using IBM SPSS Statistics version 21.0 (IBM Corp., Armonk, NY, USA).

\section{RESULTS}

\section{Subject data}

The initial study population consisted of 41 volunteers who were recruited from the patients of Seoul National University Dental Hospital. One subject withdrew consent to participate in the study, resulting in a total of 40 participants who participated in the study (Table 1). The subjects of the manual toothbrush group ranged in age from 39 to 75 years (mean \pm standard deviation [SD] $=54.5 \pm 10.6$ years) and included 11 men and nine women. The subjects of the powered toothbrush group ranged in age from 27 to 75 years (mean $\pm \mathrm{SD}=54.6 \pm 11.7$ years) and included 12 men and eight women. A total of 33 implants were present in the manual toothbrush group, and 30 implants were present in the powered toothbrush group. The time of implant restoration ranged from 2.7 to 9.0 years (mean $\pm \mathrm{SD}=5.6 \pm 2.8$ years) in the manual toothbrush group and from 3.2 to 8.1 years (mean $\pm S D=4.8 \pm 2.9$ years) in the powered toothbrush group. The time of implant placement ranged from 3.0 to
Table 1. Description of subjects and implants by toothbrush group.

\begin{tabular}{|lcc|}
\hline & \multicolumn{2}{c|}{ Toothbrush group } \\
\cline { 2 - 3 } Subject/implant characteristics & Manual & Powered \\
\cline { 2 - 3 } Gender & & 12 \\
Male & 11 & 8 \\
Female & 9 & 20 \\
Subtotal & 20 & \\
Age (year) & & $54.6(11.7)$ \\
Mean (SD) & $54.5(10.6)$ & $27-75$ \\
Range & $39-75$ & 30 \\
Number of implants & & $1.50(0.76)$ \\
Total (all subjects) & 33 & $1-3$ \\
Mean per subject (SD) & $1.65(0.75)$ & $3.2(2.5)$ \\
Range & $1-4$ & $3.6-8.7$ \\
Time since implant restoration (year) & & $4.8(2.9)$ \\
Mean (SD) & $5.6(2.8)$ & 3.1 \\
Range & $2.7-9.0$ & \\
Time since implant placement (year) & & \\
Mean (SD) & $3.0-9.6$ & \\
Range & & \\
\hline
\end{tabular}

SD: standard deviation.

9.6 years (mean $\pm \mathrm{SD}=6.4 \pm 3.1$ years) in the manual toothbrush group and from 3.6 to 8.7 years (mean $\pm S D=6.2 \pm 2.5$ years) in the powered toothbrush group.

\section{Subject oral hygiene habits and compliance}

The data collected from the questionnaire relating to oral hygiene habits and compliance are shown in Table 2. The daily frequency of brushing in the manual toothbrush group was significantly higher than in the powered toothbrush group, whereas the duration of brushing in the manual toothbrush group was significantly shorter than in the powered toothbrush group at one and two months of follow-up. The frequency of use of an interdental brush and dental floss in the manual toothbrush group was comparable to that found in the powered toothbrush group. At one month and two months of follow-up, larger differences were found between the two groups. The percentage of patient compliance was significantly different between the two groups at one month of follow-up, but not at two months.

\section{Intraexaminer and interexaminer reliability}

The intraexaminer evaluation yielded high reproducibility, with intraclass correlation coefficients for JWL and JEL of 0.941 and 0.912 , respectively. The interexaminer evaluation likewise yielded high reliability, with a kappa value for JWL and JEL of 0.91 . 
Table 2. Oral hygiene habits and compliance at baseline, one month, and two months.

\begin{tabular}{|c|c|c|c|c|c|c|}
\hline \multirow{2}{*}{ Oral hygiene habit } & \multicolumn{3}{|c|}{ Manual tooth brush } & \multicolumn{3}{|c|}{ Powered tooth brush } \\
\hline & Baseline & 1 month & 2 months & Baseline & 1 month & 2 months \\
\hline Frequency of brushing per day & $2.33(0.56)$ & $2.58(0.50)$ & $2.50(0.51)$ & $2.36(0.49)$ & $2.16(0.37)^{a)}$ & $2.20(0.41)^{\mathrm{a}}$ \\
\hline Time of brushing (minute) & $1.85(0.73)$ & $2.27(0.57)$ & $2.41(0.52)$ & $2.02(0.80)$ & $3.00(0.58)^{a)}$ & $2.82(0.48)^{a}$ \\
\hline Use of interdental brush (\%) & 63 & 67 & 63 & 56 & 40 & 48 \\
\hline Use of dental floss (\%) & 29 & 40 & 54 & 24 & 24 & 24 \\
\hline Use of water irrigation device (\%) & 0 & 0 & 0 & 0 & 0 & 0 \\
\hline Use of toothpicks (\%) & 8 & 0 & 0 & 8 & 4 & 4 \\
\hline Use of mouthwash (\%) & 13 & 13 & 8 & 24 & 12 & 12 \\
\hline Patient compliance for tooth brushing (\%) & & $95.2(4.6)$ & $96.1(6.1)$ & & $98.7(3.5)^{a)}$ & $99.5(1.1)$ \\
\hline
\end{tabular}

a)Statistically significant compared to the manual toothbrush group at the same time.

Table 3. Reduction in clinical parameters between baseline and post-baseline follow-up points.

\begin{tabular}{|c|c|c|c|c|c|c|}
\hline \multirow{2}{*}{ Clinical parameters } & \multicolumn{2}{|c|}{ Base to 1 month } & \multicolumn{2}{|c|}{1 month to 2 months } & \multicolumn{2}{|c|}{ Base to 2 months } \\
\hline & Mean \pm SD & $P$-value ${ }^{\text {b) }}$ & Mean \pm SD & $P$-value ${ }^{\mathrm{b})}$ & Mean \pm SD & $P$-value $\mathrm{e}^{\mathrm{bl}}$ \\
\hline \multicolumn{7}{|l|}{$\mathrm{mPI}$} \\
\hline Manual & $0.67 \pm 0.85$ & $<0.001$ & $0.09 \pm 0.68$ & 0.439 & $0.76 \pm 0.90$ & $<0.001$ \\
\hline Powered & $1.07 \pm 0.91$ & 0.001 & $0.20 \pm 0.71$ & 0.134 & $1.27 \pm 0.87$ & $<0.001$ \\
\hline$P$-value $e^{\text {al }}$ & 0.104 & & 0.499 & & 0.043 & \\
\hline \multicolumn{7}{|l|}{ mSBI } \\
\hline Manual & $0.48 \pm 0.91$ & 0.006 & $0.09 \pm 0.77$ & 0.491 & $0.58 \pm 0.79$ & 0.001 \\
\hline Powered & $0.63 \pm 0.61$ & $<0.001$ & $0.30 \pm 0.60$ & 0.013 & $0.93 \pm 0.87$ & $<0.001$ \\
\hline$P$-value ${ }^{\text {al }}$ & 0.300 & & 0.242 & & 0.100 & \\
\hline
\end{tabular}

${ }^{a}$ Mann-Whitney test, used for comparison between the groups.

${ }^{b}$ Wilcoxon signed rank test, used for comparison within the groups.

mPI: modified plaque index, mSBI: modified sulcus bleeding index, SD: standard deviation.

\section{Clinical parameters at the implant sites}

The $\mathrm{mPI}$ and $\mathrm{mSBI}$ scores were observed in the manual and powered toothbrush groups at baseline and at one and two months of follow-up. In the manual toothbrush group, the mean \pm SD $\mathrm{mPI}$ scores at the baseline, one month, and two months were $1.85 \pm 0.62$, $1.18 \pm 0.98$, and $1.09 \pm 0.95$, respectively. In the powered toothbrush group, the corresponding $\mathrm{mPI}$ values were $1.70 \pm 0.65,0.63 \pm 0.72$, and $0.43 \pm 0.68$, respectively. A significant difference in the $\mathrm{mPI}$ values of the manual and powered toothbrush groups was found at two months of follow-up.

An overall pattern in which $\mathrm{mPI}$ and $\mathrm{mSBI}$ values decreased postbaseline was observed in both groups (Table 3). In both groups, statistically significant reductions of $\mathrm{mPI}$ and $\mathrm{mSBI}$ values were found at one month and two months of follow-up compared to the baseline values. Moreover, in the powered toothbrush group, a statistically significant reduction in the $\mathrm{mSBI}$ was found between one and two months of follow-up (Fig. 1). The overall reductions were not significantly different between the two groups, except the $\mathrm{mPI}$ reduction between baseline and two months of follow-up (Table 3).
The Spearman coefficients between age and the change of $\mathrm{mPI}$ and $\mathrm{mSBI}$ (from baseline to two months of follow-up) were -0.209 ( $P$ value $=0.244)$ and $-0.102(P$-value $=0.508)$ in the manual toothbrush group and $-0.044(P$-value $=0.818)$ and $-0.034(P$-val$\mathrm{ue}=0.860$ ) in the powered toothbrush group, respectively.

\section{DISCUSSION}

The results from the present study have demonstrated that the use of both manual and powered toothbrushes led to significant reductions in $\mathrm{mPI}$ and $\mathrm{mSBI}$ scores. However, no significant differences were found between the two groups for any post-baseline metric except $\mathrm{mPI}$ at two months of follow up. This finding could be due to a therapeutic effect and a genetic effect. Professional scaling was performed at the first visit to minimize plaque that had accumulated around the natural teeth and the dental implant, because the presence of bacterial plaque could have affected the clinical parameters evaluated in this study. Oral hygiene education was also performed at the first visit, because the subjects had not 


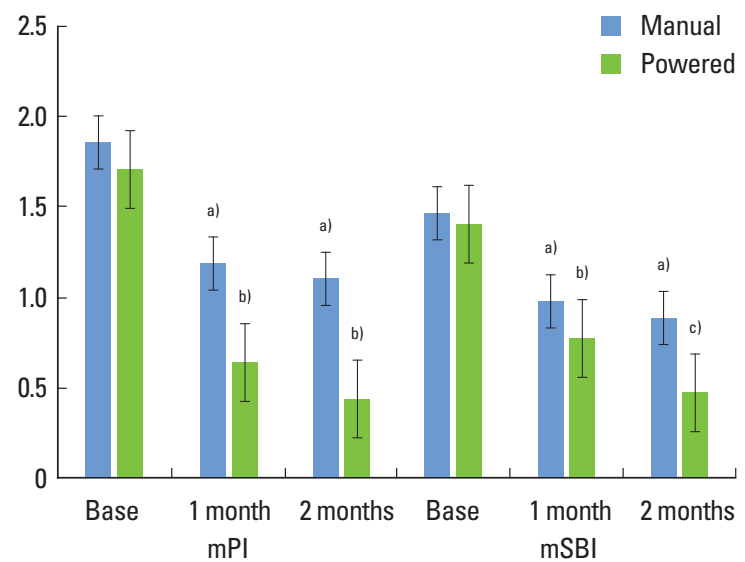

Figure 1. Clinical assessments of the modified plaque index $(\mathrm{mPI})$ and the modified sulcus bleeding index ( $\mathrm{mSBI}$ ) at the implant sites by visit and group. An overall pattern of reduction in the $\mathrm{mPI}$ and $\mathrm{mSBI}$ was observed postbaseline in both groups. In addition, in the powered toothbrush group, a statistically significant reduction of the $\mathrm{mSBI}$ was found between one month and two months of follow-up.

a) $P<0.05$ compared to the baseline in the manual toothbrush group.

b) $P<0.05$ compared to the baseline in the powered toothbrush group.

c) $P<0.05$ compared to the baseline and one month of follow-up in the powered toothbrush group.

undergone recent oral hygiene education at the same time point before the study. Professional scaling and oral hygiene education could conceal the differences between manual and powered toothbrushes in a relatively short-term study, in which the therapeutic effect could be substantial $[14,21]$. Unlike short-term studies, long-term studies have reported significant differences in gingival bleeding depending on the use of manual or powered toothbrushes $[21,22]$. Therefore, if we had investigated the subjects in a long-term study, it is probable that differences in the $\mathrm{mPI}$ or $\mathrm{mSBI}$ values would have been observed. Another factor leading to these results is the possible effect individual genetic factors on host immunity $[23,24]$. Depending on individual genetic factors, the inflammatory response of soft tissue to bacterial plaque could be different. Therefore, genetic factors could affect the variation of parameters such as $\mathrm{mSBI}$ between the two groups. A larger sample size would probably be necessary to rule out such differences.

Various types of powered toothbrush motion exist, including side to side, counter-oscillation, rotation oscillation, circular, sonic, and ionic. The powered toothbrush provided to the subjects in this study was a sonic toothbrush that uses high-velocity lateral vibrations. In their review study, Yaacob et al. [25] reported that sonic toothbrushes reduced plaque and gingivitis more than manual toothbrushes. In another review study, sonic toothbrushes were found to be inferior to rotation/oscillation-powered toothbrushes for removing plaque, especially from tooth surfaces that are difficult to clean, such as the lingual and proximal surfaces [26]. Based on previous studies and the present study, we can conclude that sonic-powered toothbrushes have a positive effect on plaque control, even if it has not been shown that they are superior to other types of powered toothbrushes.

Biofilm overgrowth in the peri-implant pocket contributes to the increase of inflammatory mediators, such as matrix metalloproteinases and interleukins. Assessing changes of both clinical and biological parameters over time would enable a more thorough evaluation of the effectiveness of manual and powered toothbrushes in peri-implant mucositis patients. In particular, cytokine levels should be evaluated in future studies, in addition to $\mathrm{mPI}$ and $\mathrm{mSBI}$ values.

Powered toothbrushes can be especially useful for geriatric patients with impaired manual skills. Verma and Bhat [27] compared the efficacy of manual and powered toothbrushes in patients aged 68-85 years. They reported that powered toothbrushes were more effective than manual toothbrushes in removing plaque and controlling gingivitis. However, in the present study, the age of the patient was not found to contribute significantly to the change of $\mathrm{mPI}$ or mSBI from baseline to two months of follow-up. Both the manual and powered toothbrush groups contained only three elderly subjects (with an age > 65 years). More elderly subjects should be recruited in future studies in order to identify the relationship between age and the change of $\mathrm{mPI}$ and $\mathrm{mSBI}$ values in response to treatment.

\section{CONFLICT OF INTEREST}

This study was supported by a grant from the Philips Corporation.

\section{ORCID}

Jungwon Lee http://orcid.org/0000-0002-5508-442X

Jong heun Lim http://orcid.org/0000-0002-8034-8976

Jungeun Lee http://orcid.org/0000-0001-8921-984X

Sungtae Kim http://orcid.org/0000-0001-6361-4104

Ki-Tae Koo http://orcid.org/0000-0002-9809-2630

Yang-Jo Seol http://orcid.org/0000-0002-2076-5452

Young Ku http://orcid.org/0000-0001-5682-0604

Yong-Moo Lee http://orcid.org/0000-0002-5619-3847

In-Chul Rhyu http://orcid.org/0000-0003-4110-6381

\section{REFERENCES}

1. Salvi GE, Aglietta M, Eick S, Sculean A, Lang NP, Ramseier CA. Reversibility of experimental peri-implant mucositis compared with experimental gingivitis in humans. Clin Oral Implants Res 2012;23:182-90.

2. Heitz-Mayfield $\sqcup$, Lang NP. Comparative biology of chronic and aggressive periodontitis vs. peri-implantitis. Periodontol 2000 2010;53:167-81.

3. Zitzmann NU, Berglundh T. Definition and prevalence of peri-implant diseases. J Clin Periodontol 2008;35:286-91.

4. Van Steenberghe $D$, Klinge $B$, Lindén U, Quirynen $M$, Herrmann I, Garpland C. Periodontal indices around natural and titanium 
abutments: a longitudinal multicenter study. J Periodontol 1993; 64:538-41.

5. Lang NP, Mombelli A, Tonetti MS, Brägger U, Hämmerle CH. Clinical trials on therapies for peri-implant infections. Ann Periodontol 1997;2:343-56.

6. Roos-Jansåker AM, Lindahl C, Renvert $H$, Renvert S. Nine- to fourteen-year follow-up of implant treatment. Part I: implant loss and associations to various factors. J Clin Periodontol 2006;33:283-9.

7. Serino G, Ström C. Peri-implantitis in partially edentulous patients: association with inadequate plaque control. Clin Oral Implants Res 2009;20:169-74.

8. Ferreira SD, Silva GL, Cortelli JR, Costa JE, Costa FO. Prevalence and risk variables for peri-implant disease in Brazilian subjects. J Clin Periodontol 2006;33:929-35.

9. Schou S, Holmstrup P, Hjørting-Hansen $E_{1}$ Lang NP. Plaque-induced marginal tissue reactions of osseointegrated oral implants: a review of the literature. Clin Oral Implants Res 1992;3:149-61.

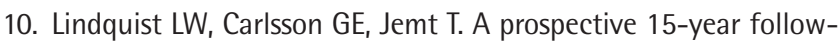
up study of mandibular fixed prostheses supported by osseointegrated implants. Clinical results and marginal bone loss. Clin Oral Implants Res 1996;7:329-36.

11. Axelsson P, Lindhe J, Nyström B. On the prevention of caries and periodontal disease. Results of a 15-year longitudinal study in adults. J Clin Periodontol 1991;18:182-9.

12. Duncan JP, Taylor TD, Kosis DL. A simple toothbrush modification for easier implant-supported prosthesis hygiene. J Prosthodont 1998;7:49-50.

13. Vandekerckhove $B$, Quirynen $M$, Warren PR, Strate J, van Steenberghe $D$. The safety and efficacy of a powered toothbrush on soft tissues in patients with implant-supported fixed prostheses. Clin Oral Investig 2004;8:206-10.

14. Sicilia A, Arregui I, Gallego M, Cabezas B, Cuesta S. A systematic review of powered vs manual toothbrushes in periodontal causerelated therapy. J Clin Periodontol 2002;29 Suppl 3:39-54.

15. Wolff L, Kim A, Nunn M, Bakdash B, Hinrichs J. Effectiveness of a sonic toothbrush in maintenance of dental implants. A prospective study. J Clin Periodontol 1998;25:821-8.
16. Swierkot $K$, Brusius $M$, Leismann D, Nonnenmacher $C$, Nüsing $R$, Lubbe $D$, et al. Manual versus sonic-powered toothbrushing for plaque reduction in patients with dental implants: an explanatory randomised controlled trial. Eur J Oral Implantol 2013;6: 133-44.

17. Bass CC. An effective method of personal oral hygiene. J La State Med Soc 1954;106:57-73.

18. Mombelli A, van Oosten MA, Schurch E Jr, Land NP. The microbiota associated with successful or failing osseointegrated titanium implants. Oral Microbiol Immunol 1987;2:145-51.

19. Assaf AV, Tagliaferro EP, Meneghim Mde C, Tengan C, Pereira AC, Ambrosano GM, et al. A new approach for interexaminer reliability data analysis on dental caries calibration. J Appl Oral Sci 2007;15:480-5.

20. Kim HY. Statistical notes for clinical researchers: Evaluation of measurement error 1: using intraclass correlation coefficients. Restor Dent Endod 2013;38:98-102.

21. Ainamo J, Xie 0 , Ainamo A, Kallio P. Assessment of the effect of an oscillating/rotating electric toothbrush on oral health. A 12-month longitudinal study. J Clin Periodontol 1997;24:28-33.

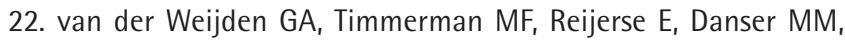
Mantel MS, Nijboer A, et al. The long-term effect of an oscillating/rotating electric toothbrush on gingivitis. An 8-month clinical study. J Clin Periodontol 1994;21:139-45.

23. Yoshie H, Kobayashi T, Tai H, Galicia JC. The role of genetic polymorphisms in periodontitis. Periodontol 2000 2007;43:102-32.

24. Michalowicz BS, Diehl SR, Gunsolley JC, Sparks BS, Brooks CN, Koertge $T E$, et al. Evidence of a substantial genetic basis for risk of adult periodontitis. J Periodontol 2000;71:1699-707.

25. Yaacob $M$, Worthington HV, Deacon SA, Deery $C$, Walmsley $A D$, Robinson $P G$, et al. Powered versus manual toothbrushing for oral health. Cochrane Database Syst Rev 2014;6:CD002281.

26. Grender J, Williams K, Walters P, Klukowska M, Reick H. Plaque removal efficacy of oscillating-rotating power toothbrushes: review of six comparative clinical trials. Am J Dent 2013;26:68-74.

27. Verma S, Bhat KM. Acceptability of powered toothbrushes for elderly individuals. J Public Health Dent 2004;64:115-7. 